Homer CSE. Using the Zelen design in randomised controlled trials: debates and controversies. Journal of Advanced Nursing. 2002, 38 (2): 200-207.

\title{
Using the Zelen design in randomised controlled trials: debates and controversies
}

Caroline SE Homer RN RM MN PhD Midwifery Consultant: Practice Development

Midwifery Practice and Research Centre

Division of Women's and Children's Health, St George Hospital, Kogarah, New South Wales, 2217, Australia and the Centre for Family Health and Midwifery, South East Health, Faculty of Nursing, Midwifery and Health at the University of Technology Sydney.

Correspondence to CSE Homer, Division of Women's and Children's Health, St George Hospital, Kogarah, New South Wales, 2217, Australia.

Tel 6129350 3298, fax 6129350 3198, email: homerc@sesahs.nsw.gov.au

Word count: Abstract: 280; text: 3980 


\begin{abstract}
Background

The use of the randomised consent design (commonly known as the Zelen design) is a controversial issue in randomised controlled trials. In the Zelen design, participants are randomly allocated prior to seeking consent. Those participants allocated to the intervention group are then approached and offered the intervention, which they can decline or accept.

Zelen first proposed the design in 1979. It has been infrequently used since this time although there are some notable exceptions in nursing, midwifery and some medical specialties.
\end{abstract}

\title{
Aim
}

This paper describes the Zelen design, including the two forms used (the single and double consent versions) and discusses the advantages and disadvantages of using such a design.

\section{Method}

An explanation of the differences between the Zelen design and a conventional randomised controlled trial is presented. In a conventional design, detailed knowledge of the alternative interventions is given to the prospective participant. The participant gives consent and is allocated to one of the groups. In a Zelen design, participants are randomly allocated and then approached and offered the group to which they were allocated. The Zelen design is used firstly, to reduce disappointment bias in the conventional consent-randomisation process and secondly, to remove subjective bias in the recruitment process.

There are concerns relating to the use of the Zelen design. These include ethical concerns relating to the timing of random allocation and consent and the collection of clinical data.

\section{Conclusion}

It is hoped that by presenting issues pertaining to the Zelen design, other nursing and midwifery researchers may be prompted to consider its use when designing clinical research. The Zelen design is controversial and debate on the merits and shortcomings is useful. This paper contributes to the ongoing debate.

Key words: Randomised controlled trial, research design, research methodology, consent, clinical research, nursing, midwifery. 


\section{Introduction}

The randomised consent design was proposed by Marvin Zelen in 1979. In the randomised consent design, participants are randomly allocated prior to seeking consent. Those participants allocated to the intervention group are then approached and offered the intervention, which they can decline or accept. The randomised consent design is also known as pre-randomisation design and post-randomisation design (Snowden et al. 1998). In this paper it is known as the 'Zelen' design.

The Zelen design has not been widely used in nursing research, although there are some notable exceptions (Cherkin et al. 1996; Steiner et al. 2001). We have recently used the Zelen design in a randomised controlled trial of a new model of midwifery care in Australia (Homer et al. 2000; Homer et al. 2001a; Homer et al. 2001b). In both designing our trial and submitting for publication, we were subjected to many of the debates and controversies surrounding the use of the Zelen design. These are presented in this paper to help clarify many of the issues and to help others who may be considering using a Zelen design.

\section{Conventional design}

The Zelen design (1979) provides an alternative sequence in the randomisation-consent process, with randomisation preceding consent. In conventional designs, prospective participants are identified, approached and asked to provide consent and are randomly allocated to (usually) one of two options (Figure 1).

In a conventional design, detailed knowledge of the alternative interventions is given to the prospective participant. The participant gives consent and is allocated to one of the groups. Detailed knowledge of the study may influence the responses of participants in trials that measure outcomes reflecting feelings and opinions (Dennis 1997). For example, negative responses may reflect a control participant's disappointment or dissatisfaction with their allocation to an experimental or control group, whereas positive responses may correspond to a treated participant's apparent loyalty to the intervention.

The effect of the consent-randomisation progression on outcomes has also been reported in other research where the unblinded nature of the trial leaves participants, who are not randomly allocated to their treatment of choice, feeling 'disappointed and demoralised' (Bradley 1993). For example, trials in Holland testing the therapeutic effectiveness of heroin provision have reported that participants allocated to the control group have been disappointed and this has affected the trial results. Disappointment also led to large scale withdrawals from the study and adversely affected the validity of the trial (Schellings et al. 1999; Hartnoll et al. 1980). 
These examples demonstrate that the use of a conventional design can mean some trials produce misleading results. Other factors also influence the results, particularly the extent to which they can be generalised to a larger population. The next section will discuss selection bias, which can mean an unrepresentative sample is recruited.

\section{Factors that influence recruiting a representative sample}

The system of recruitment-consent-randomisation followed in conventional designs appears to be objective. In reality, objectivity may not always occur and selection bias may result. Personal experience suggests that clinicians (midwives, nurses and doctors) sometimes make personal or professional judgements about which participants researchers can access for recruitment and consent. An inability to speak English, perceived low intelligence and social or emotional problems may be used as spurious reasons to keep potential participants away from researchers. Clinicians might also have their own opinions on the merits of the research. This may mean that they are reluctant for 'their' patients to be included and deny people the capacity to decide for themselves.

Busy, understaffed outpatient clinics may also contribute to the introduction of bias as the potential sample is reduced. Clinicians may lack the time to refer participants to researchers. The sense of anxiety that is often felt in these busy areas means the pressure to move people through the system, without further delay, is high. These processes are usually subtle and clinicians and researchers may be unaware they are occurring. Nonetheless, they may mean participants are removed from the sample.

Personal experience suggests that researchers can also make idiosyncratic judgements about which potential participants are approached. Certain types of people, for example, English speaking participants who are articulate, friendly or affable may be perceived by the researcher as being more likely to participate and therefore are more likely to be approached. Frequent refusal is an unpleasant experience. Therefore researchers may be more inclined to approach those participants who they perceive are more likely to participate. A rigorous, systematic protocol to ensure that all eligible people are approached in a fair and equitable manner is important to reduce this form of bias.

Socio-economic status also seems to influence recruitment and participation. Zelen (1979) suggested that researchers are more likely to approach people from high and low socio-economic groups rather than people from the middle ranges of socio-economic status. People of high socioeconomic status are more likely to be approached because their clinicians believe they will understand the research and give consent. In contrast, people of low socio-economic status are often approached as it is felt that they will leave the decision up to the doctor. This phenomenon will affect the characteristics of the eventual sample. 
The type of trial also seems to influence the type of participants who are recruited. People who consent to large prevention trials seem to be better educated, from a higher socio-economic strata, married, employed and greater users of preventative care and other medical services (Hunninghake 1987; Hunninghake et al. 1987) Conversely, more privileged and better educated individuals are discouraged from participating in some treatment trials (Thong \& Harth 1991). This means a person's characteristics influence their likelihood of being recruited. The generalisability of results from such trials may therefore be questionable unless recruiting protocols are rigorous.

The factors identified may bias results in a randomised controlled trial. The Zelen design may be able to reduce some of the biases related to use of the conventional progression of consentrandomisation. The Zelen design is discussed in the next section.

\section{The Zelen design}

In a Zelen design, participants are identified and random allocation takes place before consent is sought for the intervention. There are two versions of the Zelen design: 'single consent' and 'double consent' (Zelen 1979; 1990) In both versions, the allocations are concealed, that is, researchers are unaware as to the next allocation.

In the single consent version, participants allocated to the control group are not asked for consent, they receive standard treatment without mention of the trial. Participants allocated to the experimental group are asked whether they consent to the new treatment. If they decline they receive the standard treatment. All randomly allocated participants are included in the analysis, whether they consented or not (Figure 2).

The double consent version differs as participants allocated to the control group are asked for their consent to the standard treatment and those who decline may receive the experimental (or some other) treatment. Participants allocated to the experimental group are asked whether they consent to the new treatment. If they decline they receive the standard treatment as in the single consent version (Figure 3).

The single consent version is suitable when the experimental treatment is only available within the confines of the trial and is being compared to standard care. The double consent version is suitable when the experimental treatment is available outside the trial, or when two 'standard' treatments are compared.

In both versions the clinician and researchers know who is to be approached for consent. An assessment of outcome can however be made blind to allocation. 


\section{Use of a Zelen design}

The Zelen design appears to have been used infrequently since it was first proposed more than 20 years ago. Only a few trials are reported in the literature. Most have been in the field of cancer, (Fisher et al. 1985; Moertel et al. 1984) with a few examples from neonatalology (Bartlett et al. 1985; O'Rourke et al. 1989) and osteoarthritis (Chang et al. 1990). Three trials of continuity of midwifery care ( Homer et al 2001a; MacVicar et al. 1993; Flint et al. 1989) and two trials of continuous support during labour (Hemminki et al. 1990; Cogan \& Spinnato 1988) have also used the Zelen design. There are two recent examples in nursing, a trial of nurse-led care in an acute service (Steiner et al. 2001) and a trial of patient education for back pain (Cherkin et al. 1996). The design has also been used in two small studies of extracorporeal membrane oxygenation in newborns babies (O'Rourke et al. 1989; Bartlett et al. 1985) It was thought to be unethical in these studies to discuss a potentially life saving treatment with parents and then withhold the treatment because the patient was allocated to the control group.

More recently, a trial used the Zelen design to test the physical, social and psychological effect of contact with a stroke family care worker (Dennis et al. 1997). Participants were randomly allocated prior to consent. Those allocated to the intervention group were approached and asked to consent to follow up. Dennis (1997) justified use of the Zelen design, writing:

detailed knowledge of the trial and its exact purposes are likely to bias or influence results. Thus, responses may reflect a control subject's disappointment or dissatisfaction with not receiving a potentially beneficial treatment or a treated patient's appreciation or loyalty to those providing the treatment (p. 1077).

The Zelen design is clearly advantageous in some research, as it seems to reduce the sense of disappointment that participants allocated to the control group express. Other advantages are also outlined in the following section.

\section{Advantages of a Zelen design}

Zelen designs have been found to be advantageous in trials that have compared operations for breast cancer where conventional designs are unpopular with participants and clinicians (Zelen 1990). In surgical research, the design has been suggested as a means to overcome the difficulty recruiting participants who have a definite preference for one procedure over another (Stirrat et al. 1992). Proponents of heroin provision trials in The Netherlands have indicated that the Zelen design would be the best choice for their research as it would result in less disappointment bias, fewer dropouts and would lead to more reliable results being obtained (Schellings et al. 1999). Schellings et al (1999) suggest that the design has wider applicability, particularly when the experimental intervention is highly attractive to potential participants and when the control group receive standard treatment. 
In a conventional design, participants have a 50 per cent chance (in a one-to-one ratio) of being able to receive the intervention. In a Zelen design, when participants who have been randomly allocated to the intervention group are approached for consent, they know that they can receive the new treatment. The Zelen design is advantageous for the participant, as they know which treatment will be given before providing consent (Zelen 1979). The consent process is simplified and there is a greater likelihood of the participants having a better understanding of the intervention to which they are consenting. Researchers may be more certain that the participant is giving informed consent to the intervention. Knowing the intervention to which participants are randomly allocated to receive may also ease both information giving and decision making processes for the clinician and participant and may not compromise the patient-physician relationship.

The Zelen design may also improve rates of accrual in trials in which recruitment is difficult, as the design is weighted to include those who might otherwise have refused (Snowden et al. 1998). Using a Zelen design may encourage greater diversity (for example, in socio-economic status, cultural background) in the sample as the potential subjective nature of recruitment is removed.

In conventional trials, ideally, all consenting participants are included in an intention to treat analysis, whereas, in a Zelen design, all randomly allocated participants are included. The postrandomisation loss in conventional designs when participants withdraw because they are allocated to the arm that they least desire, is another aspect that may be reduced with the Zelen design (Zelen 1992). While this may be a benefit of using the Zelen design, there are ethical implications to consider. These are addressed later in the paper.

There may be an apparent loss of efficiency due to the expected refusal of a proportion of participants. For example, if 90 per cent of participants accept treatment, 81 participants would be needed in a conventional design as compared to 100 in a Zelen design. Zelen (1979) argues that "this loss in efficiency may be illusory" (p. 1244). Usually, only a proportion of eligible patients in an institution are approached and asked to participate in a trial. Using a Zelen design, more patients can be entered into the trial as face-to-face approaches and selection bias does not influence recruitment. The Zelen design may mean efficiency is improved over a conventional design.

\section{Criticisms of the Zelen design}

The main criticisms of the Zelen design include ethical concerns with the consent-randomisation process, its potential loss of statistical power and the routine collection of clinical data. 


\section{The process of obtaining informed consent}

The main reason for the rejection of the Zelen design appears to be because consent is not given for randomisation (Marquis \& Huston 1994; Ellenberg 1992) In most research using a Zelen design consent is obtained post-randomisation.

Informed consent has promoted much controversy in recent years, particularly in the British Medical Journal in response to the trial conducted by Dennis et al (1997). McLean (1997) cautioned against Zelen designs because of the risk of embarking down the 'slippery slope' away from one of the fundamental ethical principles, that being, respect for persons. McLean agreed that while no harm had come to the participants in the Dennis et al trial (1997), their agreement had been based on partial rather than full information and she felt that it was dangerous to believe that this was adequate. McLean (1997) also indicated that if certain types of research could not fulfill unequivocal scientific standards, it is doubtful whether they should be done in the first place. Dennis (1997) questions whether it is ethical to randomly allocate patients into trials, which because of a methodological weakness, cannot provide an answer to the main question (p.1077).

In contrast, it has been suggested that fully informed consent for randomisation can be 'needlessly cruel' for potential trial participants (Tobias \& Souhami 1993). Tobias and Souhami (1993), who describe themselves as being committed to the value of clinical trials, believe that the process of obtaining consent is traumatic for patients at a time when sensitivity is paramount. They feel that the process can damage the patient's trust in their relationship with their doctor and patients end up being more confused and uncertain about the best decision. There is also the psychological impact as the clinician enrolling the patient in the trial has to admit that they do not know whether the intervention will actually work, leading the patient to doubt the effectiveness and possibly reducing the potential benefit. These issues can lead to low recruitment and high dropout rates, particularly among participants who have given consent and are subsequently randomly allocated to the control group.

It is possible that participants are better informed when consent is sought in a Zelen design. Participants are 100 per cent certain of the treatment they will receive in a Zelen design rather being 50 per cent certain in a conventional design.

\section{Loss of statistical power}

Some researchers reject the Zelen design claiming that, in general, a number of randomly allocated participants will refuse the intervention resulting in incompatibility of the groups (Matts \& McHugh 1987; Anbar 1983; Ellenberg 1984) A loss of statistical power may also occur if a large proportion of participants randomly allocated to the intervention group decline to participate. It has been estimated that if the overall refusal rate is greater than 15 per cent, twice the number of 
patients will have to be recruited (Ellenberg 1984). If the intervention is attractive or desirable, it is likely that this dilution effect will be minimal, as only a small proportion of participants will decline. For example, low refusal rates were seen in the trials of continuous support in labour and continuity of midwifery care (Homer et al. 2001a; MacVicar et al. 1993; Hemminki et al. 1990; Flint et al. 1989; Cogan \& Spinnato 1988). Therefore, researchers need to have confidence that more than 85 per cent of participants randomly allocated to the intervention group will accept the allocation.

\section{Collection of clinical data}

The collection of clinical data from participants who decline to take part in the trial has also been reported as a concern with use of the Zelen design (Snowden et al. 1998).

Clinical data are widely collected in health care institutions for review and quality control purposes. Data are also routinely forwarded to health departments and disease registries to enable state and national reporting of health outcomes. For example, the annual report Australia's Mothers and Babies (Day et al. 1999) is based on routine data collected without consent of the participants involved. This report and others are used widely utilised to assess maternity services and health outcomes and planning new services, however, none of the 250,000 women were asked for their consent for the collection and distribution of this information. One might suggest that these participants were actually involved in an ongoing trial, that is, a trial of the effectiveness of Australian maternity service provision.

Many hospitals collect data on the clinical outcomes of their patients. Women are not asked permission for this collection. These data are mostly used for quality control purposes and peerreview audits within maternity units. Maternity units that do not collect this information would be seen as having quality assurance practices below acceptable standards.

Recent changes to legislation in Australia and elsewhere, including the United Kingdom (UK) may make the use of routinely collected clinical data more difficult to use for research purposes. In New South Wales, privacy legislation enacted in 1998 states that personal information can only be used "for the purpose for which it was collected, for a directly related purpose, for a purpose to which the individual has consented" (NSW Government 1998, Section 17). In the UK, the Data Protection Act (Acts of the UK Parliament 1998) indicates that personal data can only be processed for research purposes if they will not cause any damage or distress to the data subject. Conditions have been placed on the use of such data that may make the use of personal data in research situations more difficult without explicit consent. Both these examples demonstrate that increasingly there are more conditions associated with the use of personal data and researchers must be mindful of these issues when planning and designing their research. The Zelen design may pose additional challenges in relation to privacy legislation. 


\section{A Zelen design instead of a conventional design}

The process of choosing the most appropriate design has been addressed in research that was conducted in the United Kingdom (UK) as a part of the Extra Corporeal Membrane Oxygenation (ECMO) trial in severely ill neonates in the mid-1990's (UK Collaborative Trial Group 1996). This trial recruited mature newborns with acute and potentially irreversible respiratory failure and randomly allocated them to either ECMO, a form of respiratory support that had little unbiased evidence as to its efficacy, or to conventional management utilising ventilator support. Parents were asked to consent to this trial when they were very distressed and anxious and were given little time to make their decision. Results of the trial indicated that infants who received ECMO were more likely to survive than those who received conventional management.

A series of qualitative interviews with 37 parents of surviving infants some time later revealed startling confusion over the randomisation and consent process (Snowden et al. 1997). Many parents did not understand the nature of the trial, the process of random allocation or the need for this method of allocation.

The researchers had initially proposed a Zelen design, however this was rejected after discussions with consumer group representatives. It was felt that the potential non-disclosure in a Zelen design was unethical and that those who were not informed would feel upset or angry that they were unwitting participants in a clinical trial. It is possible that the use of a Zelen design would have reduced the confusion relating to the process around randomisation for these parents. Zelen (1979) suggested that presenting potential participants with only one option reduces the, often difficult, decision-making process and the anxiety associated with the process of random allocation. Allmark (1999) a medical ethicist has suggested that the process of obtaining informed consent in the ECMO trial had the potential to cause harm, particularly by disappointing those who ended up in the control group. Others have argued that consent for a neonatal trial is not only difficult to obtain, it is unlikely to be fully informed because of the parents' distress and the immediacy of the decision (Mason 1997). An editorial in The Lancet suggested that the Zelen design may be appropriate in these neonatal trials (Anonymous 1995). The opposing view is that parents have the right to decide how much information they receive and should therefore be able to make their own decision about participation (Meren 1995).

Conscious of this ongoing debate about the potential usefulness of the Zelen design in the ECMO trial, Snowden et al (1998) conducted an additional qualitative study with the parents of 25 infants from the original trial. This study asked parents how they would have reacted to a Zelen design. The parents were evenly divided in accepting or rejecting the design, however those who rejected the design were more likely to be from the control group. It was apparent that Zelen randomisation would not necessarily minimize the stress for those who were allocated to the intervention group as the participants felt that they would still have to make an 'impossible' 
decision. Some parents saw Zelen randomisation as a kinder approach, whereas others felt that the gathering of information as a coping strategy and access to information as a right (Snowden et al. 1998). It is possible that the results of this qualitative study might have been different if the parents were unaware of the results of the ECMO trial or if the results were reversed. It is possible that responses of the parents were mediated by the fact that ECMO infants were more likely to survive and that all parents interviewed were those of surviving infants.

\section{Conclusion}

This paper has discussed the reasons for the use of the Zelen design and presented some of the advantages and disadvantages of the design. The Zelen design provides one means to reduce systematic bias in clinical trials. It is helpful because maintains allocation concealment, potentially reduces subjective bias in the process of asking people to participate in trials and helps overcome the difficulty in recruiting people who have a definite preference for one intervention over another. It may also be useful in reducing disappointment bias, particularly in research where 'satisfaction' is an outcome.

However, there are a number of concerns raised by the use of the design. These include the need to be certain that most participants will accept the intervention offered and few people will withdraw. The other main concern is that people are randomly allocated prior to giving consent. These issues need consideration in the planning of any trial considering use of the Zelen design.

Nursing and midwifery researchers may consider using the Zelen design. However, legislation in some countries (for example, Australia and the UK) may make the Zelen design will be increasingly difficult to use. Nonetheless, the design may have applicability and utility in many settings and is worthy of consideration.

There is clearly considerable controversy over the Zelen design within the literature. Controversy, nonetheless, is healthy and necessary. Dilemmas of this nature remain open for interpretation and continued debate is required between researchers who select and use such designs as Zelen's and those who review and critique such studies. 
Figure 1: The progression of participants in a conventional randomisation design.

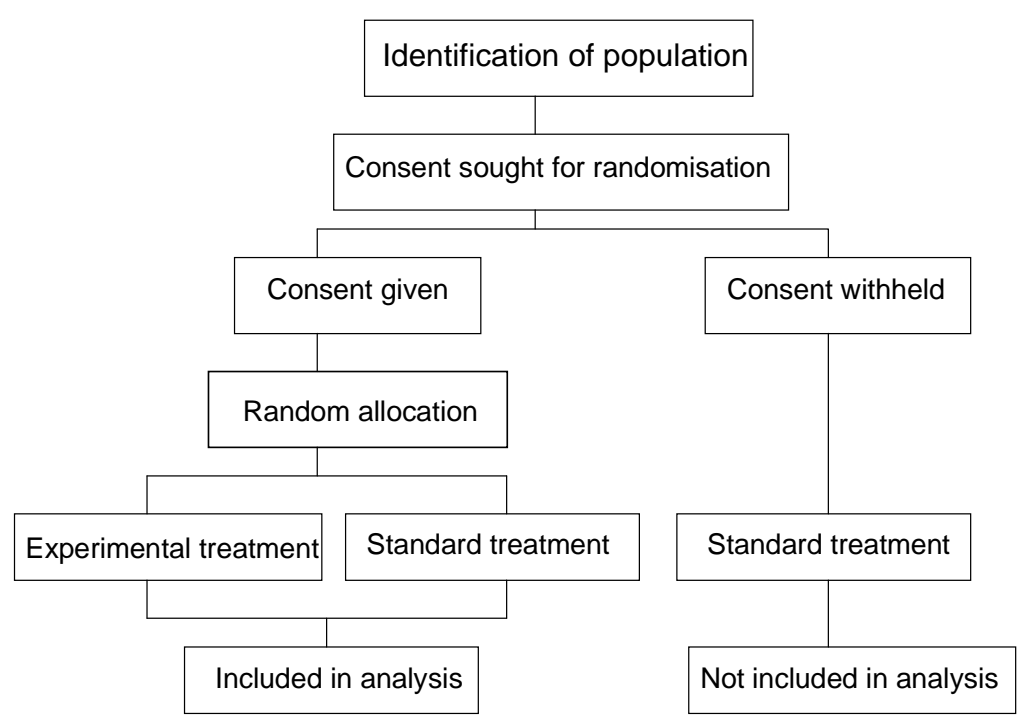


Figure 2: The progression of participants using a single consent Zelen design.

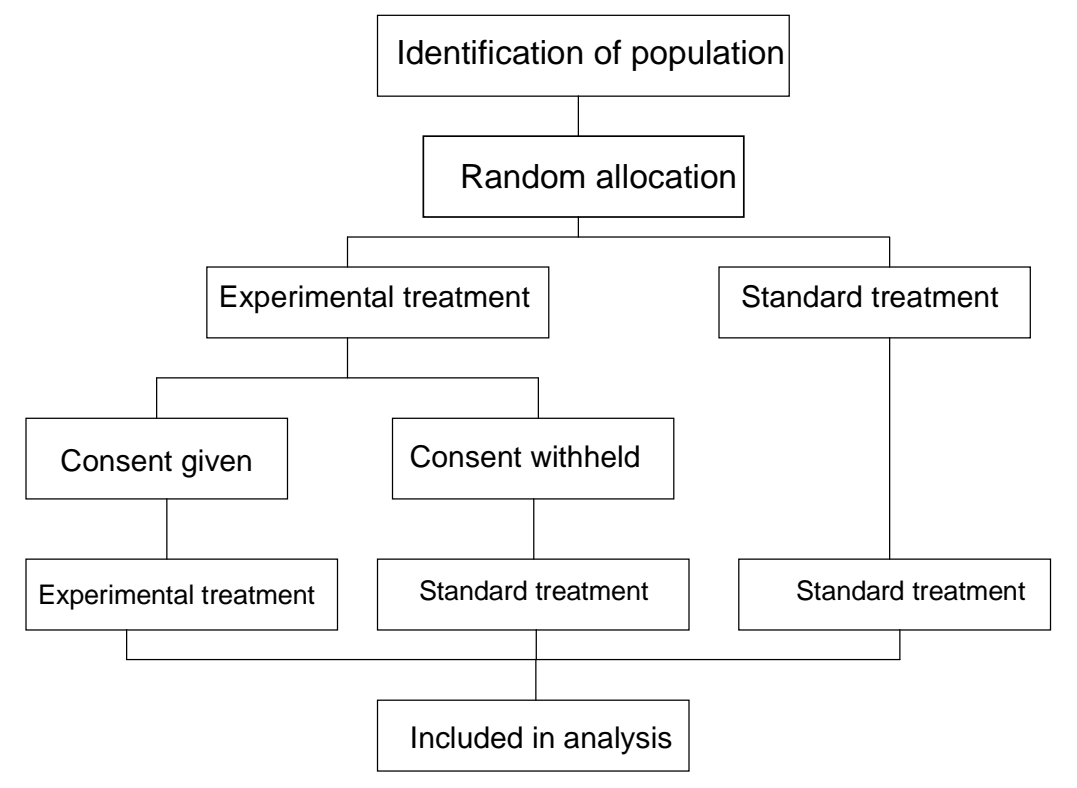


Figure 3: The progression of participants using a double consent Zelen design.

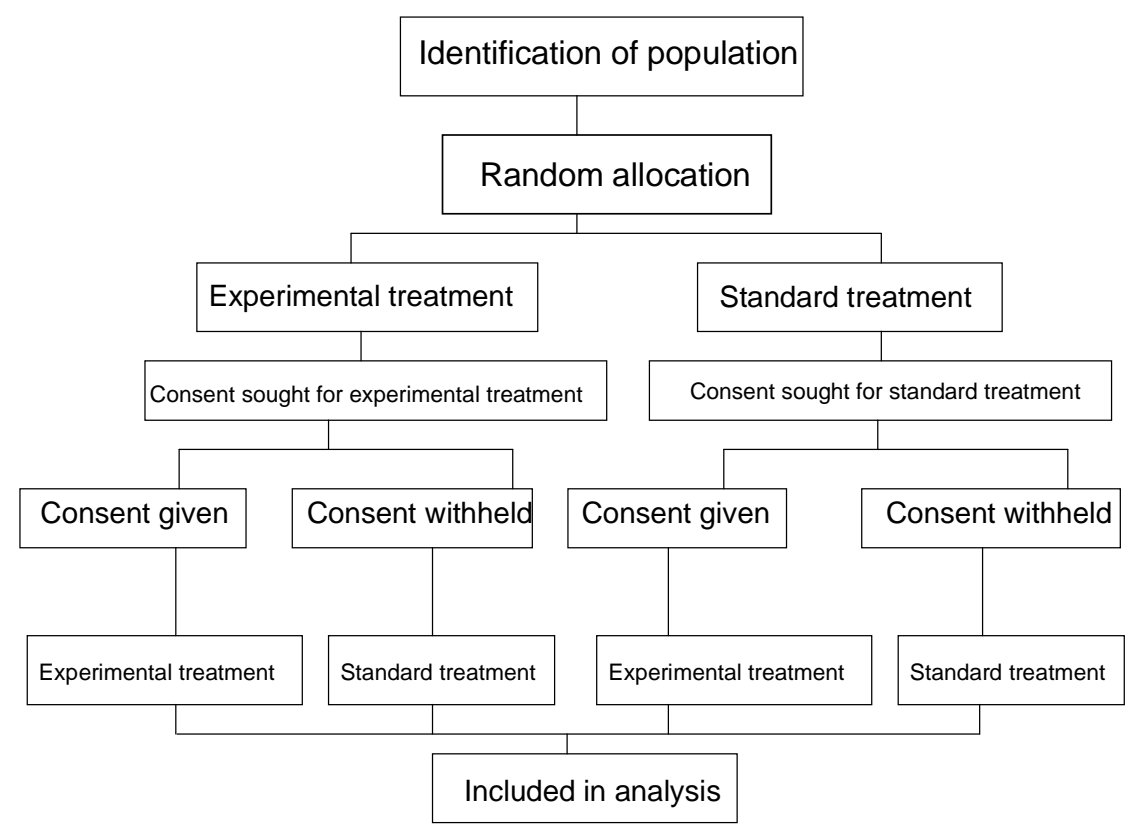




\section{References}

Acts of the UK Parliament (1998). Data Protection Act. Chapter 29 . Queen's Publisher, London.

Allmark, P. (1999). Should Zelen pre-randomised consent designs be used in some neonatal trials? Journal of Medical Ethics. 25 (4): 325-329.

Anbar, D. (1983). The relative efficiency of Zelen's pre-randomisation design for clinical trials. Biometrics. 39 (3): 711-718.

Anonymous (1995). Your baby is in a trial [editorial]. Lancet. 345 805-806.

Bartlett, R. H., Roloff, D. W., Cornell, R. G., Andrews, A. F., Dillon, O. W., Zwischenberger, S. B. (1985). Extracorporeal circulation in neonatal respiratory failure: a prospective randomised trial. Pediatrics. 76 (4): 479-487.

Bradley, C. (1993). Designing medical and educational studies. Diabetes Care. 16 (2): 509-518.

Chang, R. W., Falconer, J., Stullberg, S. D. (1990). Prerandomization: an alternative to classic randomisation. the effects on recruitment in a controlled trial of arthroscopy for osteoarthrosis of the knee. Journal of Bone Joint Surgery. 72A (10): 1451-1455.

Cherkin, D. C., Deyo, R. A., Street, J. H., Hunt, M., Barlow, W. (1996). Pitfalls of patient education: limited success of a program for back pain in primary care. Spine. 21 345-355.

Cogan, R. \& Spinnato, J. A. (1988). Social support during premature labour: effects on labour and the newborn. Journal of Psychosomatic Obstetrics \& Gynaecology. 8 209-216.

Day, P., Sullivan, E. A., Ford, J., \& Lancaster, P. (1999). Australia's mothers and babies 1997 AlHW National Perinatal Statistics Unit, Sydney.

Dennis, M. (1997). Commentary: Why we didn't ask patients for their consent. British Medical Journal. 3141077.

Dennis, M., O'Rourke, S., Slattery, J., Staniforth, T., Warlow, C. (1997). Evaluation of a stroke family care worker: results of a randomised controlled trial. British Medical Journal. 314 (7087): 1071-1076.

Ellenberg, S. S. (1984). Randomisation designs in comparative clinical trials. New England Journal of Medicine. 310 (21): 1404-1408.

Ellenberg, S. S. (1992). Randomized consent designs in comparative clinical trials: an update [letter]. Statistics in Medicine. 11 (1): 131-132.

Fisher, B., Bauer, M., Margolese, R. (1985). Five year results of a randomised controlled trial comparing total mastectomy and segmental mastectomy with or without radiation in the treatment of breast cancer. New England Journal of Medicine. 321 (11): 665-673. 
Flint, C., Poulengeris, P., Grant, A. (1989). The "Know Your Midwife' scheme - a randomised trial of continuity of care by a team of midwives. Midwifery. 5 (1): 11-16.

Hartnoll, R. L., Mitcheson, M. C., Battersby, A., Brown, G., Ellis, M., Fleming, P. (1980). Evaluation of heroin maintenance in a controlled trial. Archives of General Psychiatry. 37 (8): 877884.

Hemminki, E., Virta, A. L., Koponen, P., Malin, M., Kojo-Austin, H., Tuimala, R. (1990). A trial of continuous human support during labour: feasibility, interventions and mothers' satisfaction (Trial A - Pilot study with volunteered midwifery students). Journal of Psychosomatic Obstetrics \& Gynaecology. 11 239-250.

Homer, C. S. E., Davis, G. K., Brodie, P. (2000). What do women feel about community-based antenatal care? Australian and New Zealand Journal of Public Health. 24 (5): 590-595.

Homer, C. S. E., Davis, G. K., Brodie, P. M., Sheehan, A., Barclay, L. M., Wills, J., Chapman, M. G. (2001a). Collaboration in maternity care: a randomised controlled trial comparing communitybased continuity of care with standard care. British Journal of Obstetrics and Gynaecology. 108 (1): $16-22$.

Homer, C. S. E., Matha, D., Jordan, L. G., Wills, J., Davis, G. K. (2001b). Community-based continuity of midwifery care versus standard hospital care: a cost analysis. Australian Health Review. 24 (1): 85-93.

Hunninghake, D. B. (1987). Summary conclusions. Controlled Clinical Trials. 8 (suppl.4): 1S-5S.

Hunninghake, D. B., Darby, C. A., Probstfield, J. L. (1987). Recruitment experience in clinical trials: Literature summary and annotated bibliography. Controlled Clinical Trials. 8 (suppl.4): 6S$30 \mathrm{~S}$.

MacVicar, J., Dobbie, G., Owen-Johnstone, L., Jagger, C., Hopkins, M., Kennedy, J. (1993). Simulated home delivery in hospital: a randomised controlled trial. British Journal of Obstetrics and Gynaecology. 100 (4): 316-323.

Marquis, B. \& Huston, C. (1994). Management Decision Making for Nurses, 2nd edn, Lippincott, New York.

Mason, S. (1997). Obtaining informed consent for neonatal randomised controlled trials - "an elaborate ritual"? Archives of Diseases in Childhood. 76 (3): F143-F145.

Matts, J. \& McHugh, R. (1987). Randomization and efficiency in Zelen's single-consent design. Biometrics. 43 (4): 885-894.

McLean, S. (1997). Commentary: No consent means not treating the patient with respect. British Medical Journal. 314 1076-1077.

Meren, J. G. (1995). Ethics of consent for babies in randomised clinical trials [letter]. Lancet. 345 1370. 
Moertel, C. G., Childs, D. S., O'Fallon, J. R. (1984). Combined 5-fluoroouracil and radiation therapy as a surgical adjuvant for poor prognosis gastric carcinoma. Journal of Clinical Oncology. 2 (11): 1247-1254.

NSW Government (1998). Privacy and Personal Information Protection Act NSW State Government, Sydney. .Website: www.austlii.edu.au/au/legis/nsw/consol_act/papipa1998464

O'Rourke, P. P., Crone, R. K., Vacanti, J. P. (1989). Extracorporeal membrane oxygenation and conventional medical therapy in neonates with persistent pulmonary hypertension of the newborn: a prospective randomised study. Pediatrics. 84 (6): 957-963.

Schellings, R., Kessels, A. G. H., van Riet, G., Sturmans, F. (1999). The Zelen design may be the best choice for a heroin-provision experiment. Journal of Clinical Epidemiology. 52 (6): 503-507.

Snowden, C., Elbourne, D., Garcia, J. (1998). Zelen randomisation: Attitudes of parents participating in a neonatal clinical trial. Controlled Clinical Trials. 20 149-171.

Snowden, C., Garcia, J., Elbourne, D. (1997). Making sense of randomization; responses of parents of critically ill babies to random allocation of treatment in a clinical trial. Social Science and Medicine. 45 (9): 1337-1355.

Steiner, A., Walsh, B., Pickering, R. M., Wiles, R., Ward, J., Brooking, J. I. (2001). Therapeutic nursing or blocking beds? A randomised controlled trial of a post acute immediate care unit. British Medical Journal. 322 453-460.

Stirrat, G. M., Farndon, J., Farrow, S. C., Dwyer, N. (1992). The challenge of evaluating surgical procedures. Annals of the Royal College of Surgeons England. 74 (80): 84.

Thong, Y. H. \& Harth, S. C. (1991). The social filter of informed consent in clinical research. Pediatrics. 87 568-569.

Tobias, J. S. \& Souhami, R. L. (1993). Fully informed consent can be needlessly cruel. British Medical Journal. 307 1199-1201.

UK Collaborative Trial Group (1996). Uk collaborative randomised trial of neonatal extra-corporal membrane oxygenation. Lancet. 348 75-82.

Zelen, M. (1979). A new design for randomized controlled trials. New England Journal of Medicine. 300 (22): 1242-1245.

Zelen, M. (1990). Strategy and alternative designs for clinical trials: an update. Statistics in Medicine. 9 (6): 645-656.

Zelen, M. (1992). Randomised controlled trials [letter]. Lancet. 340 (8815): 375. 


\section{Acknowledgments}

Dr Margaret Cooke and Professor Lesley Barclay provided useful guidance on earlier versions of this manuscript. I am grateful for the advice and discussion on this debate provided from Dr Greg Davis and Professor Mark Brown at St George Hospital and for the useful comments from the two independent reviewers.

Caroline Homer was supported by an Australian National Health and Medical Research Council Centres of Excellence in Hospital-based Research Grant.

Conflict of interest: none 